The peeling behaviour of a graphene sheet on a nano-scale corrugated surface

This content has been downloaded from IOPscience. Please scroll down to see the full text.

2013 J. Phys. D: Appl. Phys. 46435305

(http://iopscience.iop.org/0022-3727/46/43/435305)

View the table of contents for this issue, or go to the journal homepage for more

Download details:

IP Address: 159.226.199.8

This content was downloaded on 12/11/2013 at 02:36

Please note that terms and conditions apply. 


\title{
The peeling behaviour of a graphene sheet on a nano-scale corrugated surface
}

\author{
Hao Chen and Shaohua Chen \\ LNM, Institute of Mechanics, Chinese Academy of Sciences, Beijing, 100190, \\ People's Republic of China \\ E-mail: chenshaohua72@hotmail.com
}

Received 25 June 2013, in final form 29 August 2013

Published 7 October 2013

Online at stacks.iop.org/JPhysD/46/435305

\begin{abstract}
The peeling process and average peeling force of a graphene (GE) sheet on a corrugated surface are investigated using molecular dynamics simulation. It is found that the peeling behaviour varies with the substrate surface roughness and the peeling angle. Three kinds of typically peeling behaviours include (a) GE sheet directly passing the valley of the substrate roughness; (b) bouncing off from the substrate; and (c) continuously peeling off similarly to that on a flat substrate. As a result, the average peeling force is strongly dependent of the peeling behaviours. Furthermore, some interesting phenomena are caught, such as partial detaching and partial sliding of GE sheet in the valley of the substrate roughness, which are mainly due to the effects of pre-tension in GE sheet and the reduction of friction resistance. The results in this paper should be useful for the design of nano-film/substrate systems.
\end{abstract}

(Some figures may appear in colour only in the online journal)

\section{Introduction}

As a special film material, graphene (GE) possesses exceptional properties, such as the extraordinary electrical [1] and thermal [2] ones, which give it great potential for many nano-scale applications, e.g. in nano-electromechanical systems. Its extremely small thickness and flexibility but with exceptional mechanical strength $[3,4]$ also make GE a good choice as a coating layer, which can protect the substrate from corrosion and oxidation, reduce the surface friction and improve the wear resistance [5]. In almost all the applications, the adhesive interface between GE and the substrate is very important for the services of devices or structures, which will show significant influence on the electronic and mechanical properties [6-8]. As a result, many interesting works have been carried out in order to understand the morphology of the adhesive interface $[9,10]$ and the interface strength [11].

For an ideal smooth substrate, full conformation morphology would be formed between GE sheet and the substrate. However, no perfectly smooth surface exists in nature, which leads to much research on the final configuration between GE and a corrugated substrate. Scharfenberg et al [10] revealed that GE undergoes a sharp 'snap-through' transition as a function of the layer thickness on a corrugated substrate, i.e., closely conforming to the substrate or remaining flat on the substrate. Wagner and Vella [12] demonstrated that substrate shapes should play a crucial role in determining the 'snapthrough' transition and proposed a substrate shape that could exhibit a continuous, rather than 'snap-through' transition. Gao and Huang [13] found that the bending modulus, which increases drastically from monolayer to multilayered GE [14], also plays an important role in the transition from conformal to non-conformal morphology of a GE membrane on a corrugated surface. Recently, the morphology of the adhesive interface between a GE sheet and a nano-scale corrugated surface was investigated by Chen et al [15], in which it was found the final configuration depends not only on the surface roughness of the substrate and the number of GE layers, but also on the length of GE; a continuous transition, rather than the 'snap-through' one was exhibited by the MD simulations, i.e., GE remaining flat on the substrate, partial conformation to the substrate and full conformation. The results [15] can be explained by the competition between the van der Waals interaction, the bending energy and the work of friction force.

Another important aspect is the interface strength. Works related to a smooth substrate are not in the discussion scope of this paper. The interface strength considering the effect of substrate roughness has recently attracted the interest of many scientists. This topic was studied initially by Fuller and Tabor [16], in which a simple model was developed with the assumption of surface asperity following a Gaussian distribution and it was found that relative small surface 


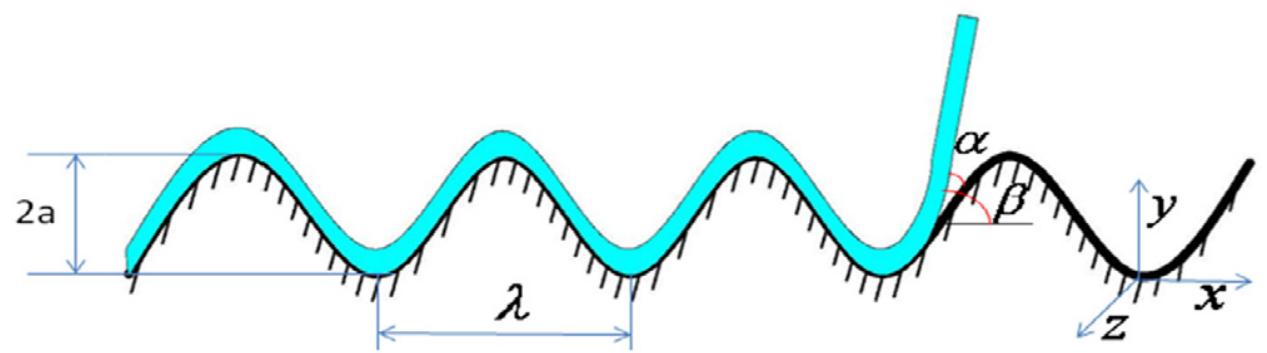

Figure 1. Schematic of a finite-size GE sheet peeling-off from a sinusoidal rough substrate. $\alpha$ is a real peeling angle at each point on the substrate surface and $\beta$ is a nominal peeling angle. $a$ and $\lambda$ are the amplitude and wavelength of the substrate roughness.

roughness could reduce or even remove adhesion. This conclusion was accepted for a long time until Briggs and Briscoe [17] found a rubber sticking a rigid slightly rough surface much better than a relatively smooth one. In order to find the mechanisms, many theoretical and numerical models considering the effect of surface roughness on adhesion were established [18-26]. It was shown that adhesion of an elastic solid to a rough substrate involves the competition between the attractive adhesion energy, which results mainly from the atomic contact at the interface, and the repulsive elastic energy, associated with the bending of the elastic solid surface. When the attractive adhesion energy is larger than the repulsive elastic energy, the elastic solid/film will deform spontaneously to fill out the substrate cavities, and the complete contact will occur [27]. Adhesion force may be improved by the surface roughness, which may explain the phenomenon found in [17]. In fact, all the theoretical adhesion models for thin films considering the effect of surface roughness are focused on films with infinite length, which should predict different mechanical phenomena for thin film with finite length scale, such as Peng et al [25] and Peng and Chen [28].

Almost all the theoretical models considering the effect of substrate roughness adopt an assumption that the adhesive interface has a full conformation configuration. However, from our former work [15], it was found three typical configurations exist between GE sheet and a corrugated substrate actually due to the substrate roughness, i.e., flat configuration, partial conformation and full conformation. What are the differences of the interface strength of such three different morphologies? What are the peeling features of such three different morphologies? Furthermore, the peeling force exhibits a vibration feature in many peeling tests and the effect of substrate roughness is thought as one of the main factors. However, the detailed effects of substrate roughness on the peeling force are not abstracted, which may be a trivial problem in macroscopic scales but non-trivial in nano-scales.

In order to answer the above questions, the peeling behaviours of a GE sheet on corrugated surfaces are investigated using molecular dynamics simulation and the adhesive interface morphologies are based on one of our former works [15].

\section{Simulation method and atomic model}

In the molecular dynamics model, where a GE sheet adheres on a copper substrate with a corrugated surface, a Cartesian coordinate $(x, y, z)$ is introduced and the $x$ axis is parallel to the zigzag direction as shown in figure 1. Without loss of generality, the surface roughness of the substrate is described by a sinusoidal function [29], which has often been used in theoretical and experimental studies on adhesion about a corrugated substrate. The profile of the corrugated surface can be described as

$$
h_{\mathrm{s}}=h_{0}+a \sin (k x) \text {, }
$$

where $h_{0}$ is a constant, $a$ is the amplitude of the roughness, $\lambda$ is the wavelength and $k=2 \pi / \lambda$ the wave number. The corrugated copper, as a representative substrate, has a thickness larger than $(2 a+3) \mathrm{nm}$ in $y$ direction with a fixed amplitude $a=0.5 \mathrm{~nm}$. Atoms at the bottom of the substrate are fixed in all the simulations and periodic boundary conditions are adopted for the substrate in $x$ and $z$ directions. The width of GE in $z$ direction is fixed as $11.2 \mathrm{~nm}$ shown in figure 1 .

MD simulations presented in this work are performed using the large-scale atomic molecular massively parallel simulator (LAMMPS) [30]. The adaptive intermolecular reactive empirical bond order (AIREBO) [31] potential is used to describe interactions among carbon atoms [32]. The cutoff distance is $2.0 \AA$ for AIREBO potential [33]. The embedded atom potential is adopted to describe the $\mathrm{Cu}-\mathrm{Cu}$ atom interactions, which is capable of describing the structural and mechanical properties of $\mathrm{Cu}$ [34]. Interactions between carbon and copper atoms are represented by the classical L-J potential, i.e., $V(r)=4 \varepsilon\left(\sigma^{12} / r^{12}-\sigma^{6} / r^{6}\right)$. Here, $r$ is the distance between two atoms, $\varepsilon$ is a parameter determining the depth of the potential well and $\sigma$ is a length scale parameter that determines the position of the minimum potential. In all our simulations, we take $\varepsilon=0.0168 \mathrm{eV}$ and $\sigma=2.2 \AA$. All simulations are run in NVT ensemble at temperature $10 \mathrm{~K}$ using a Nosé-Hoover thermostat, unless stated otherwise. The equations of motion are solved using a velocity-Verlet algorithm with a time step $1.0 \mathrm{fs}$. In order to obtain a stable morphology, the simulation system is annealed until the potential energy of the system does not change any more. Then, one end of a spring with a spring constant $0.1 \mathrm{eV} \mathrm{nm}^{-2}$ is connected to each carbon atom at the right edge of GE sheet and the other end of the spring is pulled at a velocity $v=0.01 \mathrm{~nm} \mathrm{ps}^{-1}$. Atoms at the left edge of GE remain free during our simulations.

For the convenience of analysis, two different peeling angles $\alpha$ and $\beta$ are defined as shown in figure 1 , where $\alpha$ 
(a)

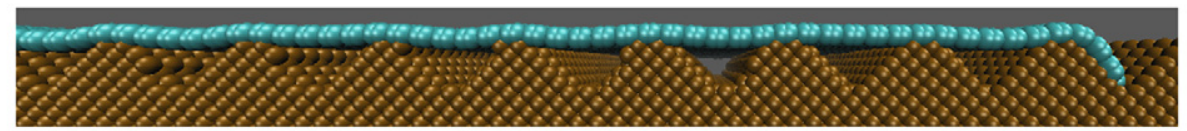

(b)

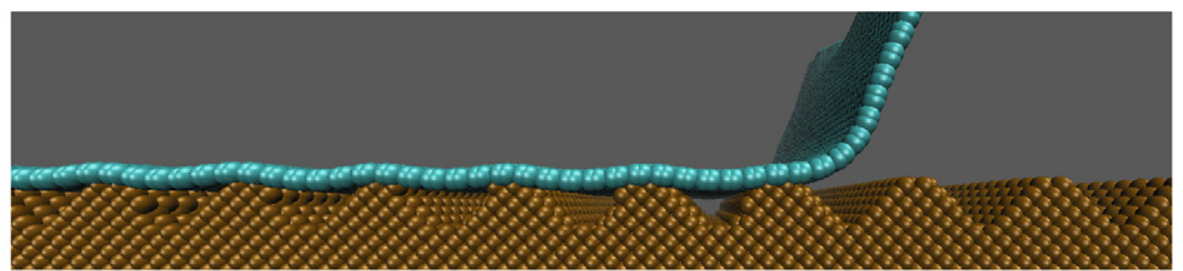

(c)

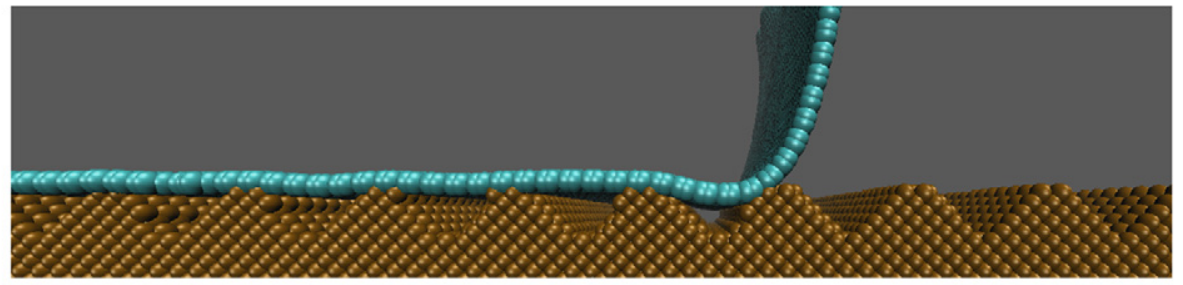

(d)

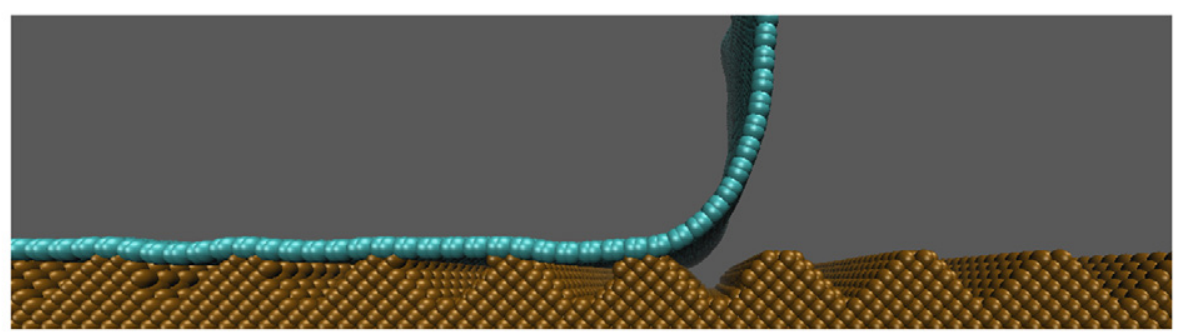

Figure 2. Side views of a GE sheet peeling-off from a corrugated substrate with $\eta=5$ and $\beta=\pi / 2$. (a) the initial adhesive morhphology; $(b)-(d)$ are snapshots of the peeling process with a time interval $150 \mathrm{ps}$

is a real peeling angle of each point on the corrugated surface and $\beta$ is a nominal peeling angle. A dimensionless parameter $\eta=\lambda / a$ is introduced in order to describe the roughness of the substrate.

\section{Results and discussions}

The effects of surface roughness and peeling angle on the peeling behaviours are mainly focused in our simulations.

Cases with a nominal peeling angle $\beta=\pi / 2$ but with different substrate roughness are investigated first, where the amplitude of the roughness is fixed as $a=0.5 \mathrm{~nm}$. Several different wavelengths of the substrate with $\eta$ ranging from 0 to 24 are chosen. During the peeling process, three kinds of different peeling behaviours with an increasing $\eta$ are found, which is significantly related to the three types of interface morphologies formed between GE and the rough substrate [15], i.e., GE sheet lying flat on a rough substrate, partial conformation between GE and rough substrate and full conformation of the adhesive interface.

The first kind of peeling behaviour is for cases with $\eta \leqslant 5$. Figure 2 gives a representative example, in which $\eta=5$ and $\lambda=2.5 \mathrm{~nm}$ lead to GE sheet lying flat on the rough surface of the substrate originally as shown in figure 2(a). Under the peeling force, GE sheet will pass directly the valley of roughness. Snapshots for GE passing one wavelength of rough substrate are given in figures $2(b)-(d)$, where the GE sheet jumps from one wave crest to another one. The relation

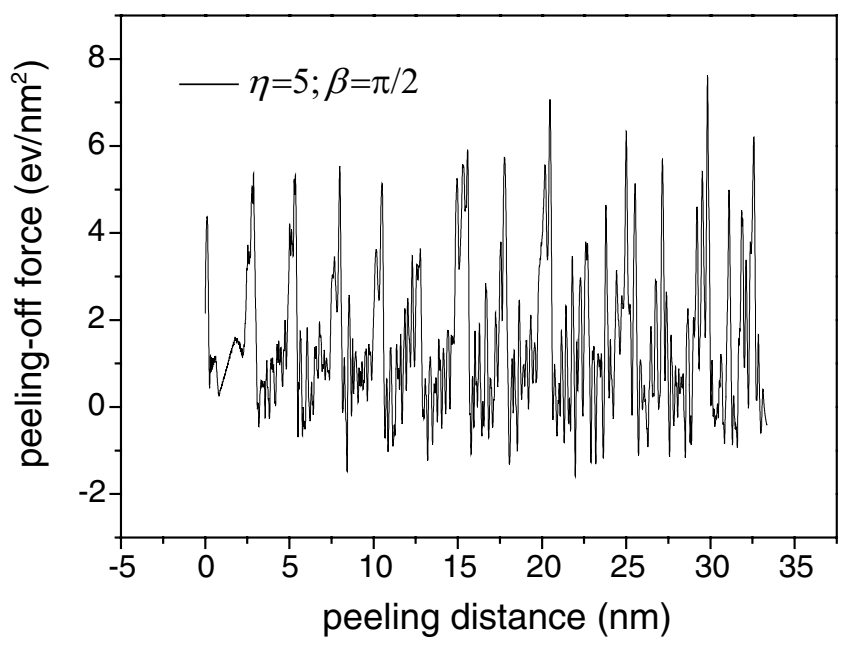

Figure 3. The peeling-off force as a function of the peeling distance for the case with $\eta=5$ and $\beta=\pi / 2$.

between the peeling force and displacement is given in figure 3, where the peeling force varies very sharply, corresponding to the adhesion region and the jumping one. The peeling force almost vanishes during the jumping stage.

Fixing the amplitude of the substrate roughness, partial conformation morphology will form between GE and rough substrate when the wavelength of the substrate roughness increases. Figure 4(a) gives an initial morphology of partial conformation, where $\lambda=3.5 \mathrm{~nm}$, i.e., $\eta=7$. During the 
(a)

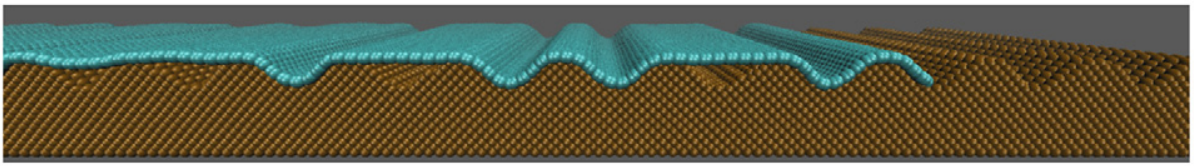

(b)

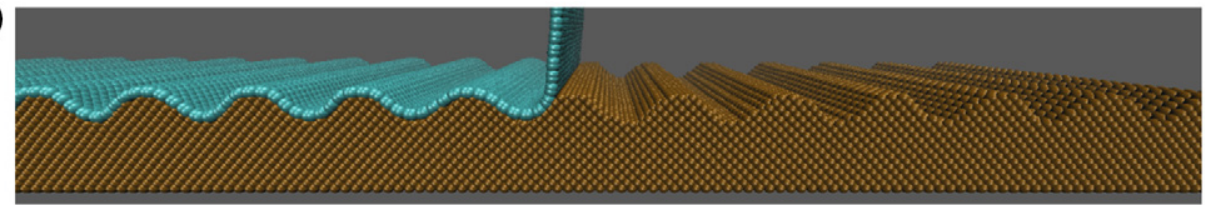

(c)

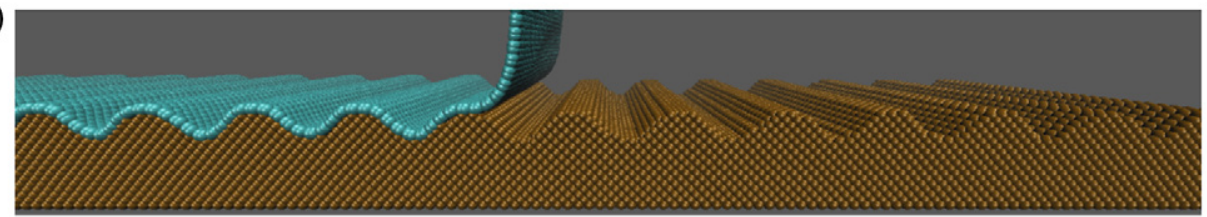

(d)

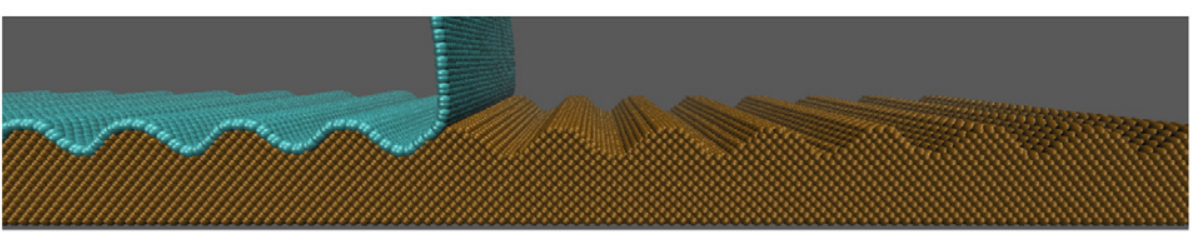

Figure 4. Side views of a GE sheet peeling-off from a corrugated substrate with $\eta=7$ and $\beta=\pi / 2$. (a) the initial adhesive morphology; $(b)-(d)$ are snapshots of the peeling process with a time interval $150 \mathrm{ps}$.

peeling process, a bouncing-off behaviour is found, which can be seen in figures $4(b)$ to $(c)$. One of the reasons is that the bending energy stored in GE is very close to the adhesion energy of the interface in this case. An additional strain energy produced by the peeling force increases a little, the total deformation energy will be larger than the adhesion energy, bouncing-off of GE from the rough substrate will happen. Another reason is that the elastic energy stored in GE increases with the gradually increasing peeling force, and simultaneously the total adhesion energy decreases due to part detachment; spontaneous bouncing-off phenomenon happens when the elastic energy is larger than the adhesion energy. Due to the bouncing-off behaviour, the peeling force descends to be a very small one that needs to straighten the loose part of GE. Meanwhile, it is interesting to find that the interface of initially partial conformation becomes full conformation during peeling, which can be found by comparing figure 4(a) to figures $4(b)-(d)$. This interesting behaviour should be reasonable and could be explained by the reduction of interface resistance due to the decreasing adhesion length of GE [15]. In other examples of initially partial conformation, for example, $\eta=6$, bouncing-off is also found and partial conformation could also become a full one. However, due to the bending energy needed in the case of $\eta=6$ larger than that in the case of $\eta=7$, the transform of partial conformation to a full one is along with the peeling process, as shown in figure 5 .

Further increasing the wavelength of the substrate roughness leads to a full conformation interface morphology [15]. Under a peeling force at the right edge of GE sheet, a continuous peeling behaviour is observed, which is similar to that in a flat surface case. Snapshots of the peeling process are shown in figure 6 with $\lambda=4.5 \mathrm{~nm}$, i.e., $\eta=9$.
Relation between the peeling force and displacement is shown in figure 7, where the peeling force varies periodically with the peeling displacement and the variation period is actually consistent with the wavelength of substrate roughness. We note that the real peeling angle $\alpha$ at each point within a wavelength is different and varies periodically.

For comparison, the peeling force in the case of a GE sheet in adhesive contact with a smooth copper substrate, i.e., $\eta=0$, is shown in figure 8 , where the peeling angle is $\beta=\pi / 2$ also. At the initial stage, the peeling force has a jump due to the unstable state, after which the peeling process enters into a stable one and the peeling force tends to be a constant. The weak fluctuation of peeling force in the flat case is attributed to the unavoidable thermo-effect during MD simulations.

Theoretical analysis is difficult for the partial conformation case, but it is easy to carry out for the full conformation case $[22,28]$. Energy conservation principle gives the energy that is needed to peel GE sheet off from substrate,

$$
E_{\mathrm{e}}=A \Delta \gamma-U_{\mathrm{el}}
$$

where $\Delta \gamma$ is the interface energy (per unit area) and takes $\Delta \gamma=0.274 \mathrm{nN} \mathrm{nm}^{-1}$ in the present work. The real contact area between GE sheet and substrate within a wavelength is $A=\int_{0}^{\lambda} \sqrt{1+\left(\partial h_{\mathrm{s}} / \partial x\right)^{2}} \mathrm{~d} x . U_{\mathrm{el}}=\frac{1}{2} D \int_{0}^{\lambda}\left(\partial^{2} h_{\mathrm{s}} / \partial x^{2}\right)^{2} \mathrm{~d} x$ is the bending energy, where $D=0.11 \mathrm{nNnm}$ is the bending modulus of one-layer GE sheet.

Then, the average peeling force can be defined as

$$
F_{\mathrm{av}}=\frac{E_{\mathrm{e}}}{L_{\mathrm{e}}},
$$


(a)

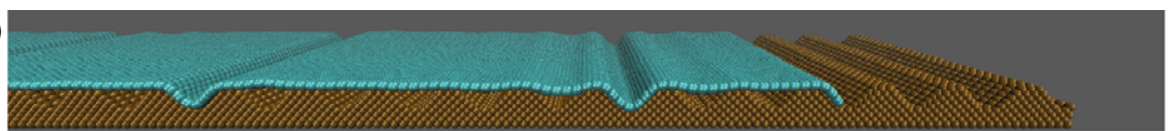

(b)

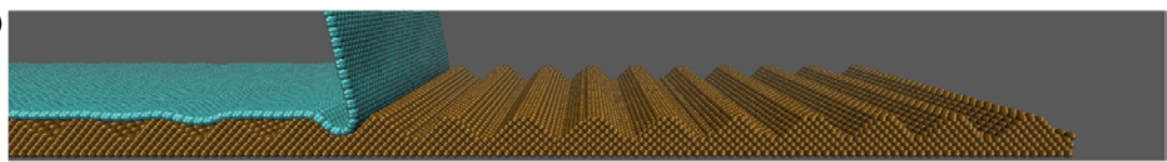

(c)

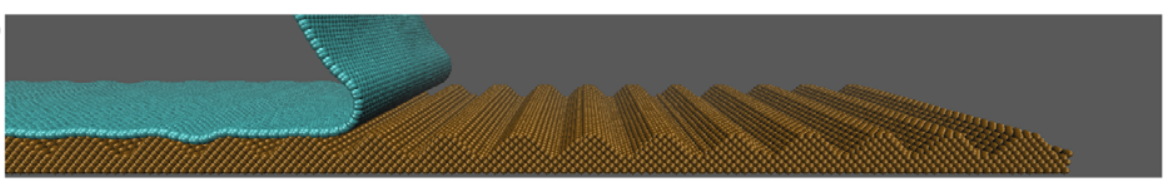

(d)

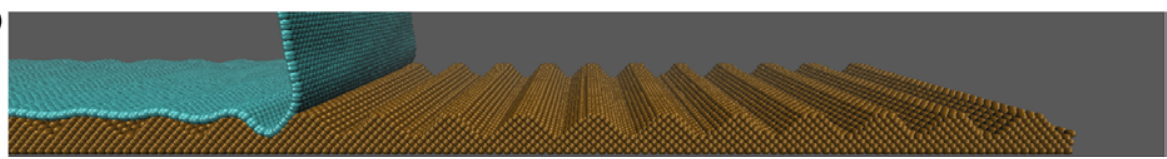

Figure 5. Side views of a GE sheet peeling-off from a corrugated substrate with $\eta=6$ and $\beta=\pi / 2$. (a) the initial adhesive morphology; (b) $-(d)$ are snapshots of the peeling process with a time interval $150 \mathrm{ps}$.

(a)

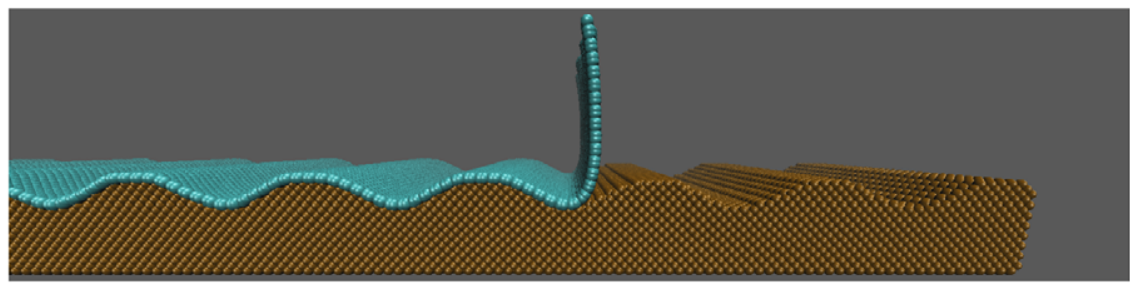

(b)

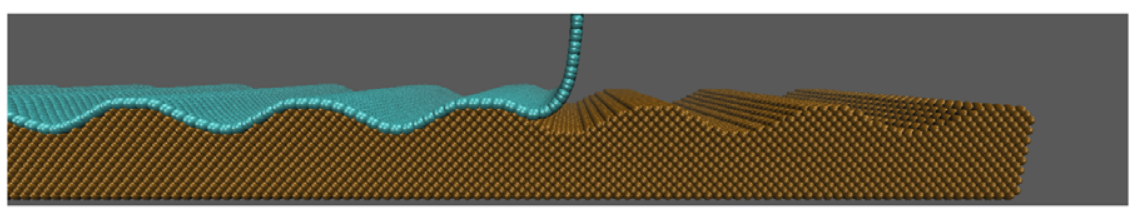

(c)

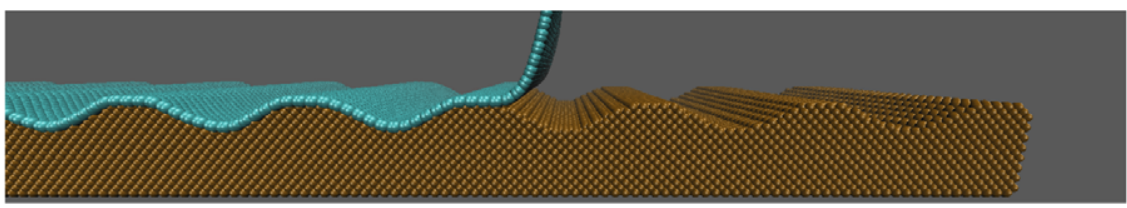

(d)

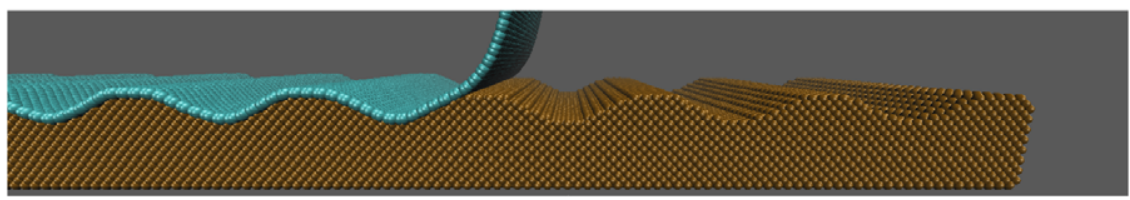

(e)

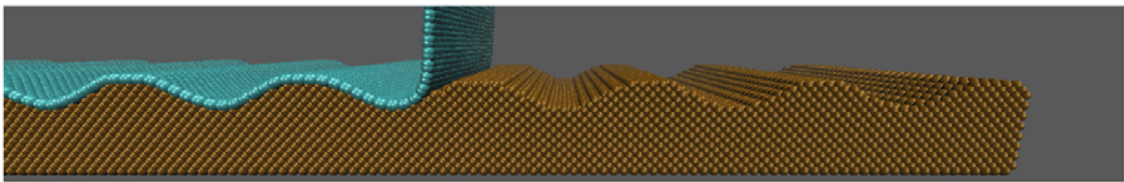

Figure 6. Side views of a GE sheet peeling-off from a corrugated substrate with $\eta=9$ and $\beta=\pi / 2$. $(a)-(e)$ are snaphsots of the peeling process with a time interval $150 \mathrm{ps}$.

where $L_{\mathrm{e}}=\int_{0}^{\lambda}(1-\cos (\alpha)) \sqrt{1+\left(\partial h_{\mathrm{s}} / \partial x\right)^{2}} \mathrm{~d} x$ is the effective peeling distance and the real peeling angle can be obtained by $\alpha=\beta-\arctan \left(\partial h_{\mathrm{s}} / \partial x\right)$.

Theoretical predictions of the average peeling force for the full conformation case are compared to the MD results with $\beta=\pi / 2$ as shown in figure 9 , where one can see that the theoretical results agree well with the simulation ones only if $\eta \geqslant 9$. Actually, in our MD models, full conformation morphology can be found when $\eta \geqslant 9$, which demonstrates the validity of energy conservation method in nano-scales. 


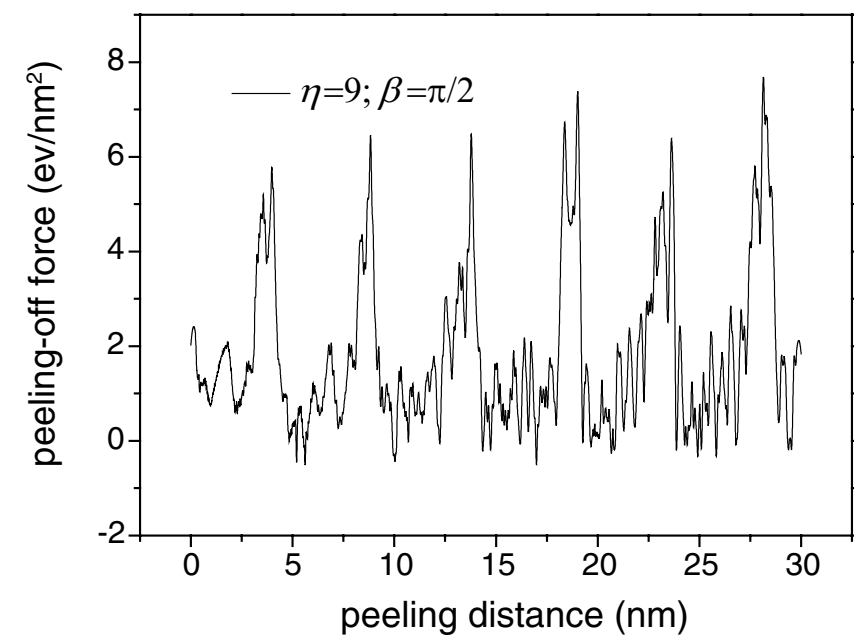

Figure 7. The peeling force as a function of the peeling distance for the case with $\eta=9$ and $\beta=\pi / 2$.

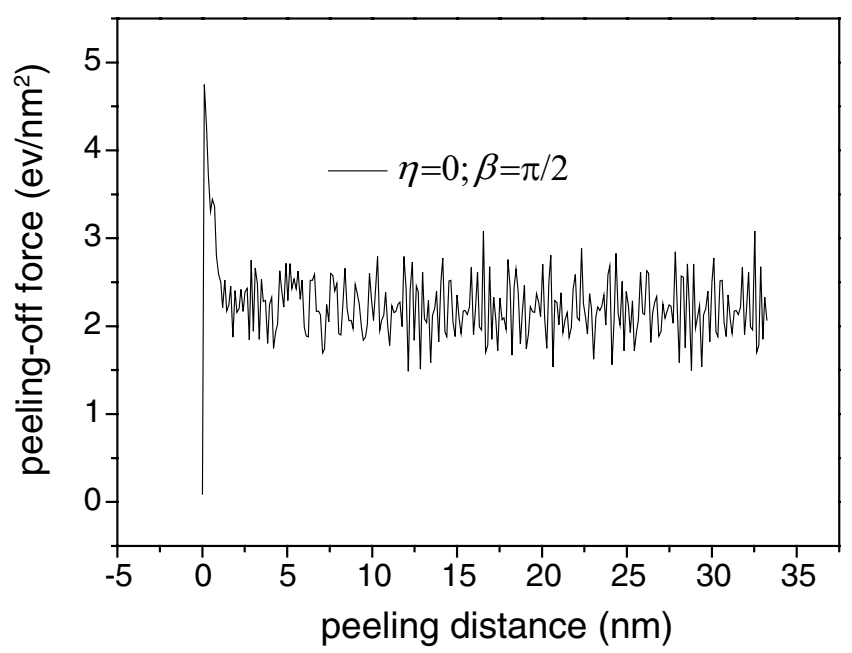

Figure 8. The peeling-off force as a function of the peeling distance for the case with a flat substrate and $\beta=\pi / 2$.

If the nominal peeling angle is not vertical to the horizontal line, do all the phenomena vary? Here, three representative cases are chosen, i.e., $\eta=5$, where GE lies flat on the corrugated substrate; $\eta=6$, where partial conformation between GE and the rough substrate forms and $\eta=9$, where full conformation can be formed. In all these cases, the peeling angle will vary in the range from $\pi / 4$ to $3 \pi / 4$.

In the case of $\eta=5$ with either a small peeling angle $\beta$ or a large one, the peeling process is almost the same as those shown in figure 2 , where $\beta=\pi / 2$ and GE sheet passes the valley of the rough substrate directly.

In the case of $\eta=6$, the peeling behaviour is influenced significantly by the peeling angle. When $\beta \leqslant \pi / 3$, a bouncingoff behaviour can be found at the beginning as shown in figures $10(a)$ and $(b)$. Then, GE sheet is stretched to lie flat on the corrugated substrate as shown in figure $10(c)$, after which the peeling process is very similar to that in the case of GE lying flat on the substrate as shown in figures $10(c)-(e)$. For the case with a small peeling angle $(\beta \leqslant \pi / 3)$, the component of peeling force in the horizontal direction is large comparing to the case with a large peeling angle, so that

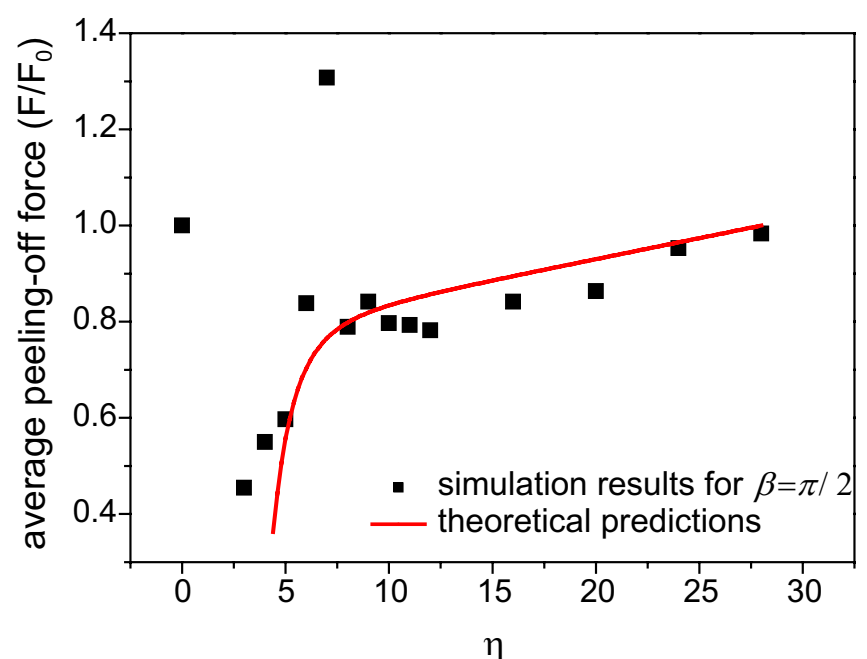

Figure 9. The average peeling-off force as a function of $\eta$, where $F_{0}$ is the corresponding peeling-off force for the flat substrate case.

GE sheet would be stretched. The stretching energy would overcome the adhesion energy, which results in GE lying flat on the corrugated substrate. When $\beta \geqslant 3 \pi / 4$, bouncing-off behaviour still exists as shown from figures $11(b)$ to $(c)$, after which the peeling force will straighten the loose part of GE and then peel off the remaining part as shown in figures 11(c) and $(d)$. When the peeling angle lies in the intermediate region, all the peeling behaviours are similar to the case of $\beta=\pi / 2$.

When $\eta=9$ but with a small peeling angle, for example $\beta \leqslant \pi / 3$, the horizontal component of the peeling force will lead to GE sliding first on the substrate and then gradually detaching from the substrate as shown in figures 12(a)-(c) [35]. For the case with a large peeling angle, i.e., $\beta \geqslant \pi / 3$, the peeling behaviours are similar to that in the case with $\beta=\pi / 2$ as shown in figure 6.

The average peeling-off force $F$ as a function of the peeling angle $\beta$ is obtained by MD simulations as shown in figure 13 for three cases with different initial interface adhesive morphologies. It is shown that the varying tendency of the average peeling-off force with the peeling angle is not influenced by the different interface morphologies. The average peeling-off force monotonically decreases with an increasing peeling angle $\beta$, which is well consistent with the finite element simulation results [36].

Finally, three more sets of simulations for three types of roughness i.e., $\eta=5, \eta=7$ and $\eta=12$ at $T=50,100$ and $300 \mathrm{~K}$, with a peeling angle $\beta=\pi / 2$ are carried out in order to investigate the effect of system temperatures on the interface peeling behaviour. It is found that the effect of system temperature on the peeling behaviour is not obvious, similar to that of a GE sheet peeled-off from a flat substrate [37]. Three kinds of typically peeling behaviours, such as the continuous peel-off, bouncing off from the substrate and directly passing the valley of the substrate roughness, are still found for models with different system temperatures. The average peeling-off forces at different system temperatures are also similar to that at $T=10 \mathrm{~K}$. As a conclusion, the peeling behaviour is mainly determined by the competition among the adhesion energy ( $\mathrm{L}-\mathrm{J}$ interaction between GE and copper), the bending 
(a)

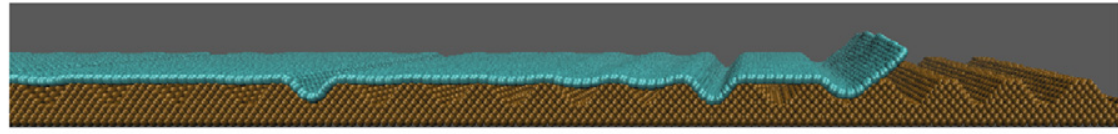

(b)

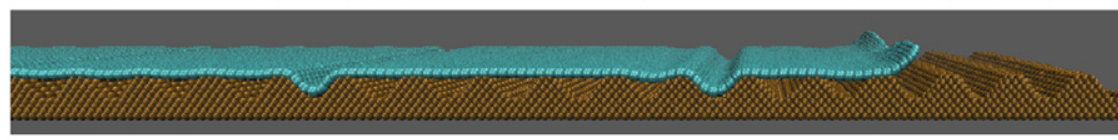

(c)

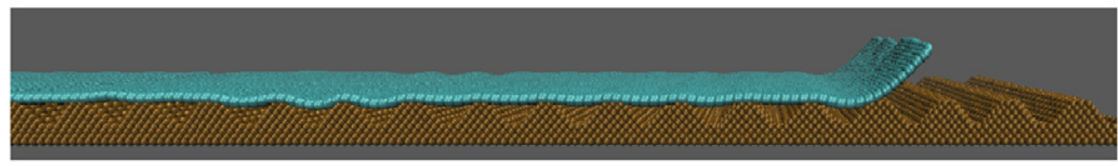

(d)

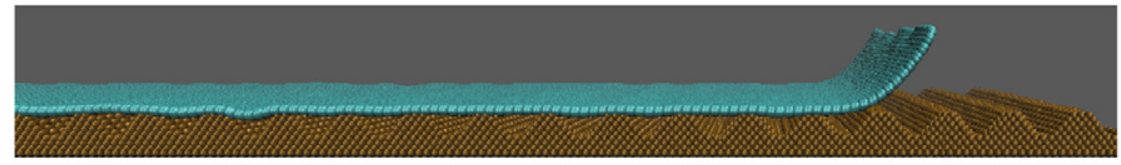

(e)

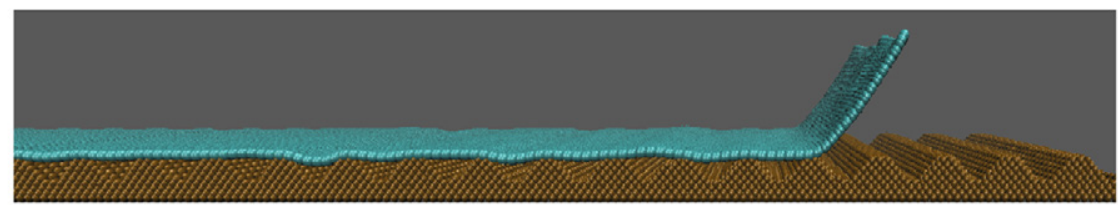

Figure 10. Side views of a GE sheet peeling-off from a corrugated substrate with $\eta=6$ and $\beta=\pi / 4$. $(a)-(e)$ are snapshots of the peeling process with a time interval $150 \mathrm{ps}$.

(a)

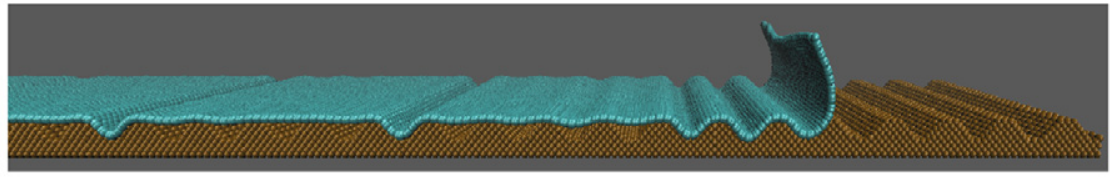

(b)

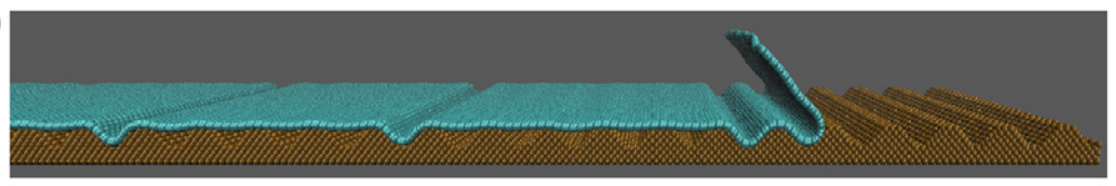

(c)

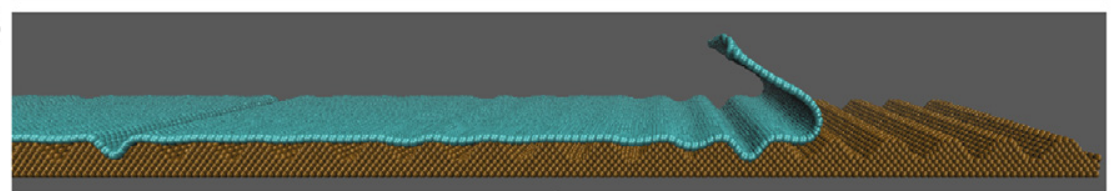

(d)

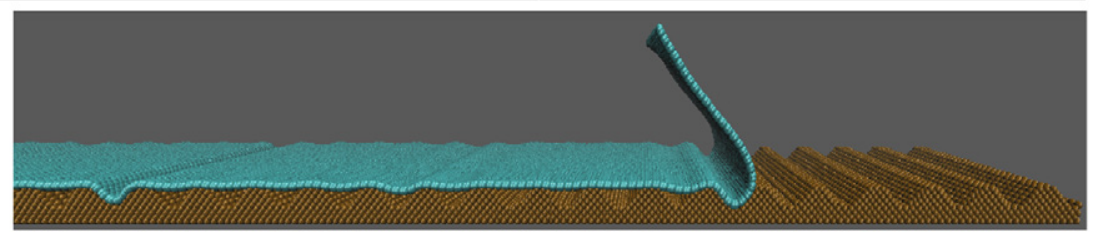

Figure 11. Side views of a GE sheet peeling-off from a corrugated substrate with $\eta=6$ and $\beta=3 \pi / 4$. $(a)-(d)$ are snapshots of the peeling process with a time interval $150 \mathrm{ps}$.

and stretching energy stored in the GE sheet and the energy consumed by the interface friction. However, the system temperature will show some effects on the free-standing GE sheet, which is peeled off from the substrate. The amplitude of vibration of the free-standing GE sheet is found to increase with an increasing system temperature.

\section{Conclusions}

In this paper, the peeling behaviour of a graphene sheet in adhesive contact with a corrugated copper substrate is investigated using molecular dynamics simulations. The peeling behaviour depends significantly on the initially adhesive interface morphology, which is mainly influenced by the roughness of the substrate and the length of GE sheet [15]. Three kinds of morphologies correspond to three kinds of peeling behaviours under a nominally vertical peeling angle: (a) GE sheet directly passing the valley of the roughness; (b) bouncing off from the substrate; (c) continuously peelingoff similar to that of a flat substrate case. When the peeling angle is small, the horizontal component of the peeling force will induce partial sliding or partial detachment and sometimes, 
(a)

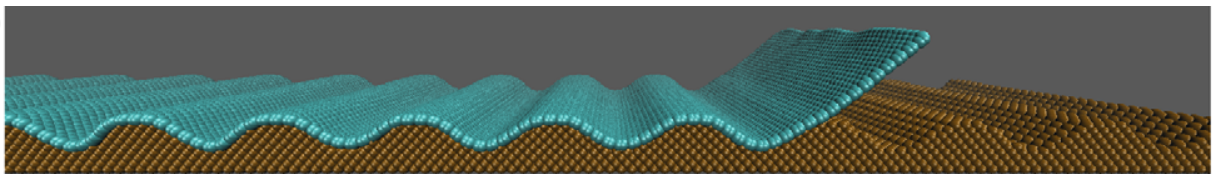

(b)

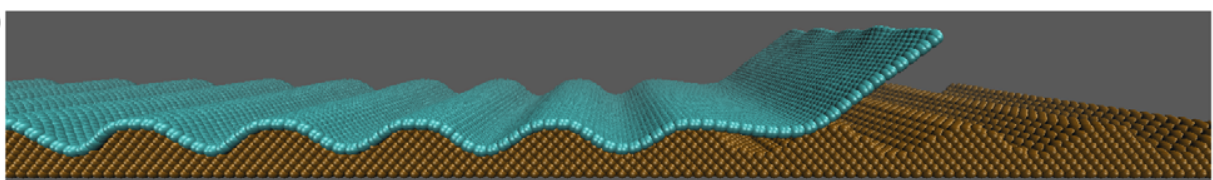

(c)

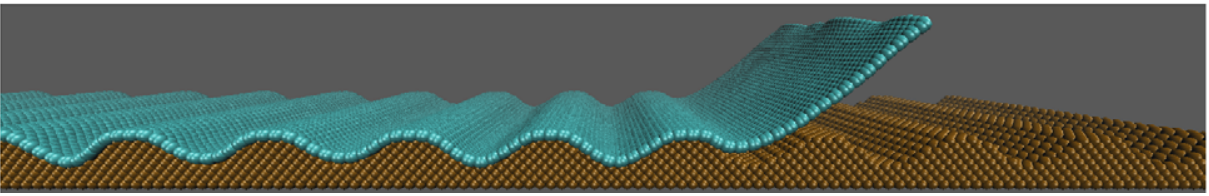

Figure 12. Side views of a GE sheet peeling-off from a corrugated substrate with $\eta=9$ and $\beta=\pi / 4$. $(a)-(c)$ are snapshots of the peeling process with a time interval $150 \mathrm{ps}$.

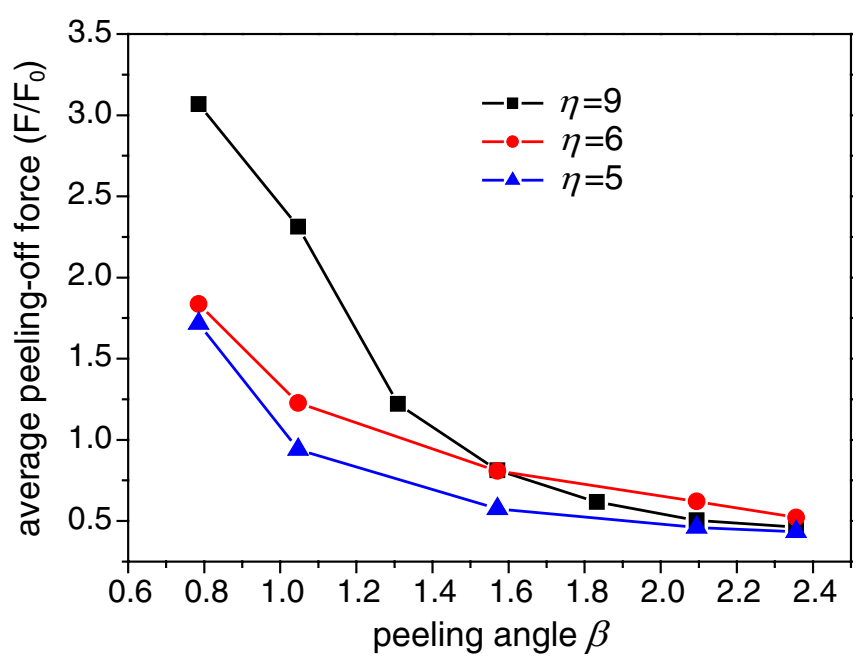

Figure 13. The average peeling-off force as a function of the peeling angle $\beta$, where $F_{0}$ is the corresponding peeling-off force for the flat substrate case.

part of the full conformation degrading to be the lying flat morphology. The peeling-off force is found to be sensitive to the peeling angle in all cases, which is consistent with the theoretical prediction for flat substrate cases $[25,35]$. The results in this paper would be helpful for the understanding of a nano-film detaching from a corrugated substrate and the design of coating on a rough substrate.

\section{Acknowledgments}

The work reported here is supported by NSFC through Grants \#11372317, \#11125211, \#11021262 and the Nano-project (2012CB937500). We are also very grateful for the helpful discussion with Xinghua Shi and Qifang Yin both from Institute of Mechanics, Chinese Academy of Science.

\section{References}

[1] Novoselov K S, Geim A K, Morozov S V, Jiang D, Katsnelson M I, Grigorieva I V, Dubonos S V and Firsov A A 2005 Nature 438 197-200
[2] Balandin A A, Ghosh S, Bao W Z, Calizo I, Teweldebrhan D, Miao F and Lau C N 2008 Nano Lett. 8 902-7

[3] Bunch J S, van der Zande A M, Verbridge S S, Frank I W, Tanenbaum D M, Parpia J M, Craighead H G and McEuen P L 2007 Science 315 490-3

[4] Lee C, Wei X D, Kysar J W and Hone J 2008 Science $321385-8$

[5] Prasai D, Tuberquia J C, Harl R R, Jennings G K and Bolotin K I 2012 ACS Nano 6 1102-8

[6] Ferrari A C et al 2006 Phys. Rev. Lett. 97187401

[7] Ishigami M, Chen J H, Cullen W G, Fuhrer M S and Williams E D 2007 Nano Lett. 7 1643-8

[8] Stolyarova E, Rim K T, Ryu S M, Maultzsch J, Kim P, Brus L E, Heinz T F, Hybertsen M S and Flynn G W 2007 Proc. Natl Acad. Sci. USA 104 9209-12

[9] Neek-Amal M and Peeters F M 2012 Phys. Rev. B 85195445

[10] Scharfenberg S, Mansukhani N, Chialvo C, Weaver R L and Mason N 2012 Appl. Phys. Lett. 100021910

[11] Koenig S P, Boddeti N G, Dunn M L and Bunch J S 2011 Nature Nanotechnol. 6 543-6

[12] Wagner T J W and Vella D 2012 Appl. Phys. Lett. 10023311

[13] Gao W and Huang R 2011 J. Phys. D: Appl. Phys. 44452001

[14] Zhang D B, Akatyeva E and Dumitrica T 2011 Phys. Rev. Lett. 106255503

[15] Chen H, Yao Y and Chen S 2013 J. Phys. D: Appl. Phys. 46205303

[16] Fuller K N G and Tabor D 1975 Pro. R. Soc. Lond. A $345327-42$

[17] Briggs G A D and Briscoe B J 1977 J. Phys. D: Appl. Phys. 102453

[18] Briggs G A D and Briscoe B J 1976 Nature $260313-5$

[19] Rabinovich Y I, Adler J J, Ata A, Singh R K and Moudgil B M 2000 J. Colloid Interface Sci. 232 10-6

[20] Rabinovich Y I, Adler J J, Ata A, Singh R K and Moudgil B M 2000 J. Colloid Interface Sci. 232 17-24

[21] Persson B N J and Tosatti E 2001 J. Chem. Phys. 115 5597-610

[22] Persson B N J 2002 Phys. Rev. Lett. 89245502

[23] Palasantzas G and De Hosson J T M 2003 Phy. Rev. E 67021604

[24] Palasantzas G and De Hosson J T M 2003 J. Appl. Phys. 94 3041-4

[25] Peng Z L, Chen S H and Soh A K 2010 Int. J. Solids Struct. 47 1952-60

[26] Greenwood J A and Williams J B 1966 Proc. R. Soc. Lond. A 295 300-19

[27] Persson B N J, Albohr O, Tartaglino U, Volokitin A I and Tosatti E 2005 J. Phys.: Condens. Matter 17 R1 
[28] Peng Z L and Chen S H 2011 Phys. Rev. E 83051915

[29] Chung S, Lee J H, Moon M W, Han J and Kamm R D 2008 Adv. Mater. 20 3011-6

[30] Plimpton S 1995 J. Comput. Phys. 117 1-19

[31] Stuart S J, Tutein A B and Harrison J A 2000 J. Chem. Phys. 112 6472-86

[32] Zhao H and Aluru N R 2010 J. Appl. Phys. 108064321
[33] Belytschko T, Xiao S P, Schatz G C and Ruoff R S 2002 Phys. Rev. B 65235430

[34] Foiles S M, Baskes M I and Daw M S 1986 Phys. Rev. B 33 7983-91

[35] Gent A N and Kaang S Y 1987 J. Adhes. 24 173-81

[36] Zhao H P, Wang Y, Li B W and Feng X Q 2013 Int. J. Appl. Mech. 51350012

[37] Shi X H, Yin Q F and Wei Y J 2012 Carbon 50 3055-63 